\title{
Analytic properties of the Virasoro modular kernel
}

\author{
Nikita Nemkov ${ }^{1,2,3, a}$ \\ ${ }^{1}$ Moscow Institute of Physics and Technology (MIPT), Dolgoprudny, Russia \\ ${ }^{2}$ Institute for Theoretical and Experimental Physics (ITEP), Moscow, Russia \\ ${ }^{3}$ The Laboratory of Superconducting metamaterials, National University of Science and Technology MISIS, Moscow, Russia
}

Received: 14 December 2016 / Accepted: 22 May 2017 / Published online: 2 June 2017

(C) The Author(s) 2017. This article is an open access publication

\begin{abstract}
On the space of generic conformal blocks the modular transformation of the underlying surface is realized as a linear integral transformation. We show that the analytic properties of conformal block implied by Zamolodchikov's formula are shared by the kernel of the modular transformation and illustrate this by explicit computation in the case of the one-point toric conformal block.
\end{abstract}

\section{Contents}

1 Introduction and results $\ldots \ldots \ldots \ldots$

2 Modular kernel . . . . . . . . . . . . . . . . . . . 2

2.1 Notation . . . . . . . . . . . . . . 2

2.2 Explicit formula . . . . . . . . . . . 2

3 Proof of the residue formula . . . . . . . . . . 3

3.1 Normalization factor . . . . . . . . . . . . 3

3.2 Regular part . . . . . . . . . . . . . 4

3.2.1 Expansion near $\alpha=\alpha_{r, s} \ldots \ldots \ldots .4$

3.2.2 Expansion near $\alpha=\alpha_{r,-s} \ldots \ldots \ldots .4$

3.2 .3 Comparison . . . . . . . . . . 5

4 Discussion . . . . . . . . . . . 6

5 Double gamma and sine functions . . . . . . . . . 6

References . . . . . . . . . . . . . . 7

\section{Introduction and results}

Conformal blocks (CBs) can be defined as universal parts of the holomorphically factorized CFT correlation functions [1]. They are recognized as a new independent class of special functions relevant for many problems in modern physics including gauge theories [2]. This paper is concerned with properties of the toric Virasoro one-point conformal block which is hereafter referred to simply as conformal block.

a e-mail: nnemkov@gmail.com
This special representative of CBs is in some sense the simplest one, although it captures a lot of the important properties present in its more sophisticated counterparts such as the spheric Virasoro blocks, $W_{N}$ - and WZW-conformal blocks, superconformal blocks etc.

The toric CB is naturally defined as the following trace:

$B_{\Delta}(q)=\operatorname{Tr}_{\Delta}\left(q^{L_{0}-\frac{c}{24}} V_{\Delta_{e}}\right)$.

Here $q$ is the toric nome $q=e^{2 \pi i \tau} ; V_{\Delta_{e}}$ is the primary field of dimension $\Delta_{e}$, the external dimension; $\Delta$ is the internal dimension - the dimension of the Verma module over which the trace is taken; and finally $c$ is the central charge of the theory. We will suppress the parameters $\Delta_{e}$ and $c$ in our notation. Definition (1) allows one to compute CB as a series expansion in powers of $q$,

$B_{\Delta}(q)=q^{\Delta-\frac{c}{24}}\left(1+q \frac{\Delta_{e}\left(\Delta_{e}-1\right)}{2 \Delta}+O\left(q^{2}\right)\right)$.

In the present paper we only consider conformal blocks with generic values of parameters. Then the $q$-expansion coefficients at arbitrary order are not known in a simple closed form. ${ }^{1}$ Nevertheless, some non-perturbative aspects of $\mathrm{CB}$ are developed. In particular, it is well known that as a function of the internal dimension $\Delta$ the conformal block has only simple poles located at the Kac zeros $\Delta=\Delta_{r, s}(11)$ and that the $q$-dependence of the corresponding residues is described by the CBs with specific external dimensions $\Delta=\Delta_{r,-s}$ (note that these are not the Kac zeros)

$\operatorname{Res}_{\Delta=\Delta_{r, s}} B_{\Delta}(q)=R_{r, s} B_{\Delta_{r,-s}}(q)$

\footnotetext{
${ }^{1}$ However, various complementary representations exist. As examples we mention the AGT-inspired representation via the Nerkasov functions [2-4] and the expansion in terms of the global conformal blocks [5], which are basically the hypergeometric functions.
} 
where $R_{r, s}$ are certain explicit $q, \Delta$-independent multipliers (12). It is also possible to find the regular part of $\mathrm{CB}$ and extend (3) to arbitrary $\Delta[6-10]$

$B_{\Delta}(q)=\chi_{\Delta}(q)+\sum_{r, s \geq 1} \frac{R_{r, s}}{\Delta-\Delta_{r, s}} q^{\Delta-\Delta_{r, s}} B_{\Delta_{r,-s}}(q)$

where $\chi_{\Delta}(q)=q^{\Delta-\frac{c-1}{24}} / \eta(q)$ is the Virasoro character. ${ }^{2}$ Interestingly, this equation provides a recurrent relation among $q$-expansion coefficients and can be used to compute $\mathrm{CB}$ order by order in $q$ without reference to the definition (1).

Another non-perturbative property of $\mathrm{CB}$ is related to the modular transformations acting on the torus and generated by the $S: \tau \rightarrow-1 / \tau$ and $T: \tau \rightarrow \tau+1$ moves. Invariance of the correlation functions together with the linear independence of CBs with different $\Delta\left(B_{\Delta}(q) \sim q^{\Delta}\right)$ imply that $S$ and $T$ are represented as linear integral transformations on the space of CBs. The $T$ transformation acts simply as a phase shift and will not be considered while the $S$ transformation is non-trivial. Denoting the kernel of the $S$-transformation by $M_{\Delta \Delta^{\prime}}$ one writes

$B_{\Delta}(q)=\int_{\Delta^{\prime}} M_{\Delta \Delta^{\prime}} B_{\Delta^{\prime}}(\widetilde{q})$

where $\widetilde{q}=q^{-2 \pi i / \tau}$. Note that the lhs and the rhs in (5) are defined as expansions in $q$ about different points and hence one cannot study this modular transformation perturbatively in $q$. Instead, the full $q$-dependence must be taken into account.

With these arrangements in place we can describe the main result of the present paper. We note that the analytic structure of conformal block (3) implies the same analytic structure for the modular kernel. Indeed, taking the residue of (5) at $\Delta=\Delta_{r, s}$ and using (3) we obtain

$R_{r, s} B_{\Delta_{r,-s}}(q)=\int_{\Delta^{\prime}} \operatorname{Res}_{\Delta=\Delta_{r, s}} M_{\Delta \Delta^{\prime}} B_{\Delta^{\prime}}(\widetilde{q})$

In turn, the conformal block $B_{\Delta_{r,-s}}(q)$ can itself be expanded via the modular transformed blocks

$B_{\Delta_{r,-s}}(q)=\int_{\Delta^{\prime}} M_{\Delta_{r,-s} \Delta^{\prime}} B_{\Delta^{\prime}}(\widetilde{q})$.

Comparing the two above equations and making use of the linear independence of CBs with different $\Delta^{\prime}$ one discovers that

$$
\operatorname{Res}_{\Delta=\Delta_{r, s}} M_{\Delta \Delta^{\prime}}=R_{r, s} M_{\Delta_{r,-s} \Delta^{\prime}} \text {. }
$$

This equation represents a non-trivial constraint required by consistency of the CB analytic structure and modular proper-

${ }^{2} \eta(q)$ is the Dedekind eta function $\eta(q)=q^{1 / 24} \prod_{n \geq 1}\left(1-q^{n}\right)$. ties. In the remainder of the text we explicitly check relation (8) to find complete agreement.

\section{Modular kernel}

\subsection{Notation}

We start by defining our notation. It is useful to introduce the Liouville-type variables $\alpha, \alpha^{\prime}, \mu, b$ replacing the original CFT data

$$
\begin{aligned}
c & =1+6 Q^{2}, \quad Q=b+b^{-1}, \\
\Delta & =Q^{2} / 4-\alpha^{2}, \quad \Delta^{\prime}=Q^{2} / 4-\alpha^{\prime 2}, \quad \Delta_{e}=\mu(Q-\mu) .
\end{aligned}
$$

With a little abuse of notation we will use the same letters for functions of the original and the newly introduced variables. Note, however, that, due to a non-trivial Jacobian of the transformation from $\Delta$ to $\alpha$ property, (8) is slightly different in terms of $\alpha$, namely

$\operatorname{Res}_{\alpha=\alpha_{r, s}} M_{\alpha \alpha^{\prime}}=-\frac{R_{r, s}}{2 \alpha_{r, s}} M_{\alpha_{r,-s} \alpha^{\prime}}$

The Kac zeros are described by

$\Delta_{r, s}=Q^{2} / 4-\alpha_{r, s}^{2}, \quad \alpha_{r, s}=\frac{r b+s b^{-1}}{2}$

for $r, s \geq 1$. We emphasize that there are no poles in $\mathrm{CB}$ at $\Delta_{r,-s}$ for $r, s \geq 1$. Note, however, that $\Delta_{r, s}=\Delta_{-r,-s}$. Without loss of generality throughout this paper we assume that $r, s \geq 1$. The multipliers entering (3) read

$R_{r, s}=A_{r, s} P_{r, s}$

where

$$
\begin{aligned}
A_{r, s}= & \frac{1}{2} \prod_{\substack{n=1-r \\
(n, m) \neq(0,0),(r, s)}}^{r} \prod_{m=1-s}^{s} \frac{1}{n b+m b^{-1}}, \\
P_{r, s} & =\prod_{n=1-r}^{r} \prod_{m=1-s}^{s}\left(n b+m b^{-1}-\mu\right) .
\end{aligned}
$$

\subsection{Explicit formula}

The modular kernel for the toric Virasoro blocks is known in closed form as an integral [11] or a series [12] representation. For our current purposes the most handy form is the following:

$$
M_{\alpha \alpha^{\prime}}=\frac{V_{\alpha}}{V_{\alpha^{\prime}}} n_{\alpha^{\prime}} \mathcal{M}_{\alpha \alpha^{\prime}}
$$


where $V_{\alpha}$ is a convenient renormalization function ${ }^{3}$

$V_{\alpha}=\frac{\Gamma_{b}(Q+2 \alpha) \Gamma_{b}(Q-2 \alpha)}{\Gamma_{b}(\mu+2 \alpha) \Gamma_{b}(\mu-2 \alpha)}$

$n_{\alpha^{\prime}}$ is an $\alpha$-independent factor

$n_{\alpha^{\prime}}=e^{2 \pi i \mu \alpha^{\prime}} \sin 2 \pi b \alpha^{\prime} \sin 2 \pi b^{-1} \alpha^{\prime} / S_{b}(\mu)$,

irrelevant for property (10), while $\mathcal{M}_{\alpha \alpha^{\prime}}$ is an essential contribution,

$$
\begin{aligned}
\mathcal{M}_{\alpha \alpha^{\prime}} & =K_{\alpha \alpha^{\prime}}+K_{-\alpha, \alpha^{\prime}}, \\
K_{\alpha \alpha^{\prime}} & =e^{4 \pi i \alpha \alpha^{\prime}} \sum_{n, m \geq 0} e^{8 \pi i \alpha_{n, m} \alpha^{\prime}} K_{n m}(\alpha), \\
K_{n m}(\alpha) & =\frac{S_{b}\left(2 \alpha+2 \alpha_{n, m}+\mu\right) S_{b}\left(2 \alpha_{n, m}+\mu\right)}{S_{b}\left(2 \alpha+2 \alpha_{n+1, m+1}\right) S_{b}^{\prime}\left(2 \alpha_{n+1, m+1}\right)} .
\end{aligned}
$$

Here $S_{b}^{\prime}(z)$ denotes the derivative of $S_{b}(z)$. Now everything is set up and we can proceed with the proof of Eq. (10).

\section{Proof of the residue formula}

We will prove assertion (10) by an explicit computation which appears to be straightforward but tedious. We would like to outline the important steps beforehand. Definition (14) represents a modular kernel as the product of the normalization factor $V_{\alpha} / V_{\alpha^{\prime}}$ (15) and the non-trivial series $\mathcal{M}_{\alpha \alpha^{\prime}}$ (17). It turns out that the normalization factor $V_{\alpha}$ features poles exactly at the Kac zeros and furthermore it satisfies

$\underset{\alpha=\alpha_{r, s}}{\operatorname{Res}} V_{\alpha}=-\frac{R_{r, s}}{2 \alpha_{r, s}} V_{\alpha_{r,-s}}$.

In contrast, the remainder $\mathcal{M}_{\alpha \alpha^{\prime}}$ appears to be regular at $\alpha=\alpha_{r, \pm s}$ and to satisfy

$\mathcal{M}_{\alpha_{r, s}, \alpha^{\prime}}=\mathcal{M}_{\alpha_{r,-s}, \alpha^{\prime}}$.

Combined, these properties lead to (10). In the rest of this section we show that Eqs. (19) and (20) hold.

\subsection{Normalization factor}

Let us compute the residue of $V_{\alpha}$ at $\alpha=\alpha_{r, s}$ and the value at $\alpha=\alpha_{r,-s}$. For $r, s \geq 1$, which we assume without loss of generality, there is a single singular multiplier in $V_{\alpha}$ at $\alpha=\alpha_{r, s}$, while at $\alpha=\alpha_{r,-s}$ everything is regular (for a summary of the analytic properties of $\Gamma_{b}(z)$ see Appendix 5). Note also that $\alpha_{r, s}+\alpha_{n, m}=\alpha_{r+n, s+m}$ and $Q=2 \alpha_{1,1}$.

\footnotetext{
3 The double gamma $\Gamma_{b}$ and sine $S_{b}$ functions to be extensively used below are described in Appendix 5.
}

Therefore, one writes

$$
\begin{aligned}
\operatorname{Res}_{\alpha=\alpha_{r, s}} V_{\alpha} & =\frac{\Gamma_{b}\left(2 \alpha_{r+1, s+1}\right) \operatorname{Res}_{\alpha=\alpha_{r, s}} \Gamma_{b}(Q-2 \alpha)}{\Gamma_{b}\left(\mu+2 \alpha_{r, s}\right) \Gamma_{b}\left(\mu+2 \alpha_{-r,-s}\right)}, \\
V_{\alpha_{r,-s}} & =\frac{\Gamma_{b}\left(2 \alpha_{r+1,1-s}\right) \Gamma_{b}\left(2 \alpha_{1-r, s+1}\right)}{\Gamma_{b}\left(\mu+2 \alpha_{r,-s}\right) \Gamma_{b}\left(\mu+2 \alpha_{-r, s}\right)} .
\end{aligned}
$$

The ratio reads

$$
\begin{aligned}
& \begin{array}{c}
\operatorname{Res} V_{\alpha} \\
\frac{\alpha=\alpha_{r, s}}{V_{\alpha_{r,-s}}}
\end{array} \\
& =\underbrace{\frac{\operatorname{Res}_{\alpha=\alpha_{r, s}} \Gamma_{b}(Q-2 \alpha)}{\Gamma_{b}\left(2 \alpha_{r+1,1-s}\right)}}_{R_{1}} \underbrace{\frac{\Gamma_{b}\left(2 \alpha_{r+1, s+1}\right)}{\Gamma_{b}\left(2 \alpha_{1-r, s+1}\right)}}_{R_{2}} \underbrace{\frac{\Gamma_{b}\left(\mu+2 \alpha_{r,-s}\right)}{\Gamma_{b}\left(\mu+2 \alpha_{-r,-s}\right)}}_{R_{3}} \\
& \quad \times \underbrace{\frac{\Gamma_{b}\left(\mu+2 \alpha_{-r, s}\right)}{\Gamma_{b}\left(\mu+2 \alpha_{r, s}\right)}}_{R_{4}} .
\end{aligned}
$$

Let us calculate the first factor

$$
\begin{aligned}
R_{1} & =\frac{\operatorname{Res}_{\alpha=\alpha_{r, s}} \Gamma_{b}(Q-2 \alpha)}{\Gamma_{b}\left(2 \alpha_{r+1,1-s}\right)}=\lim _{\epsilon \rightarrow 0} \frac{-\epsilon}{2} \frac{\Gamma_{b}\left(\alpha_{1-r, 1-s}+\epsilon\right)}{\Gamma_{b}\left(2 \alpha_{r+1,1-s}+\epsilon\right)} \\
& =\lim _{\epsilon \rightarrow 0} \frac{-\epsilon}{2} \prod_{n=1-r}^{r} \frac{\Gamma\left(n b^{2}+1-s+b \epsilon\right)}{\sqrt{2 \pi} b^{n b^{2}+1 / 2-s+\epsilon}} \\
& =\lim _{\epsilon \rightarrow 0} \frac{-\epsilon}{2} \frac{\Gamma(1-s+b \epsilon)}{\sqrt{2 \pi} b^{1 / 2-s}} \prod_{\substack{n=1-r \\
n \neq 0}}^{r} \frac{\Gamma\left(n b^{2}+1-s+\epsilon\right)}{\sqrt{2 \pi} b^{n b^{2}+1 / 2-s+\epsilon}} \\
& =\frac{(-1)^{s}}{2 \sqrt{2 \pi} b^{3 / 2-s}(s-1) !} \prod_{\substack{n=1-r \\
n \neq 0}}^{r} \frac{\Gamma\left(n b^{2}+1-s\right)}{\sqrt{2 \pi} b^{n b^{2}+1 / 2-s}} .
\end{aligned}
$$

Here the difference equation on the double gamma function (54) was used.

Computation of the second factor is more straightforward as there is no limiting procedure involved,

$R_{2}=\frac{\Gamma_{b}\left(2 \alpha_{r+1, s+1}\right)}{\Gamma_{b}\left(2 \alpha_{1-r, s+1}\right)}=\prod_{n=1-r}^{r} \frac{\sqrt{2 \pi} b^{n b^{2}+1 / 2+s}}{\Gamma\left(n b^{2}+1+s\right)}$.

Multiplying $R_{1}$ by $R_{2}$ one obtains

$$
\begin{aligned}
R_{1} \cdot R_{2} & =\frac{(-1)^{s} b^{2 s-1}}{2(s-1) ! s !} \prod_{\substack{n=1-r \\
n \neq 0}}^{r} b^{2 s} \frac{\Gamma\left(n b^{2}+1-s\right)}{\Gamma\left(n b^{2}+1+s\right)} \\
& =\frac{(-1)^{s} b^{2 s-1}}{2(s-1) ! s !} \prod_{\substack{n=1-r \\
n \neq 0}}^{r} \prod_{\substack{n=1-s \\
n=0}}^{s} \frac{1}{n b+m b^{-1}}
\end{aligned}
$$




$$
\begin{aligned}
& =\frac{(-1)^{s} b^{2 s-1}}{2(s-1) ! s !} \prod_{\substack{m=1-s \\
m \neq 0}}^{s} m b^{-1} \prod_{\substack{n=1-r \\
(n, m) \neq(0,0)}}^{r} \prod_{\substack{m=1-s \\
n}}^{s} \frac{1}{n b+m b^{-1}} \\
& =-\frac{1}{2} \prod_{\substack{n=1-r \\
(n, m) \neq(0,0)}}^{s} \prod_{\substack{m=1-s \\
n b+m b^{-1}}}^{s} \frac{1}{n b+(25)}
\end{aligned}
$$

which is exactly equal to $-\frac{A_{r, s}}{2 \alpha_{r, s}}$ with $A_{r, s}$ defined in (13). In a very similar manner one shows that the product of the remaining factors $R_{3} \cdot R_{4}$ is equal to $P_{r, s}$ defined in (13). Therefore, we conclude that Eq. (19) is satisfied.

\subsection{Regular part}

In order to prove (10) it remains to show that $\mathcal{M}_{\alpha \alpha^{\prime}}$ is regular at $\alpha=\alpha_{r, \pm s}$ and satisfies (20). Care must be taken here since the function $K_{\alpha \alpha^{\prime}}$ (18) is singular at these points, but the sum of $K_{\alpha \alpha^{\prime}}$ and $K_{-\alpha \alpha^{\prime}}$ defining $\mathcal{M}_{\alpha \alpha^{\prime}}$ turns out to be regular.

\subsubsection{Expansion near $\alpha=\alpha_{r, s}$}

Let us expand $\mathcal{M}_{\alpha \alpha^{\prime}}$ near $\alpha=\alpha_{r, s}$,

$$
\begin{aligned}
\mathcal{M}_{\alpha_{r, s}+\frac{\epsilon}{2}, \alpha^{\prime}}=e^{-4 \pi i \alpha_{r, s}} & \\
& \times\left(e^{8 \pi i \alpha_{r, s} \alpha^{\prime}} e^{2 \pi i \epsilon \alpha^{\prime}} K_{\alpha_{r, s}+\frac{\epsilon}{2}, \alpha^{\prime}}+e^{-2 \pi i \epsilon \alpha^{\prime}} K_{-\alpha_{r, s}-\frac{\epsilon}{2}, \alpha^{\prime}}\right) \\
= & e^{-4 \pi i \alpha_{r, s}} \sum_{n, m} e^{8 \pi i \alpha_{n, m} \alpha^{\prime}} \mathcal{M}_{n, m}^{r, s}(\epsilon)
\end{aligned}
$$

where we have denoted

$$
\begin{aligned}
\mathcal{M}_{n, m}^{r, s}(\epsilon)= & e^{2 \pi i \epsilon \alpha^{\prime}} K_{n-r, m-s}\left(\alpha_{r, s}+\frac{\epsilon}{2}\right) \delta_{n \geq r, m \geq s} \\
& +e^{-2 \pi i \epsilon \alpha^{\prime}} K_{n, m}\left(\alpha_{-r,-s}-\frac{\epsilon}{2}\right) .
\end{aligned}
$$

Consider

$$
\begin{aligned}
& K_{n-r, m-s}\left(\alpha_{r, s}+\frac{\epsilon}{2}\right) \\
& =\frac{S_{b}\left(2 \alpha_{n, m}+\mu+\epsilon\right) S_{b}\left(2 \alpha_{n-r, m-s}+\mu\right)}{S_{b}\left(2 \alpha_{n+1, m+1}+\epsilon\right) S_{b}^{\prime}\left(2 \alpha_{n-r+1, m-s+1}\right)} .
\end{aligned}
$$

Taking into account that the function $S_{b}\left(2 \alpha_{n+1, m+1}+\epsilon\right)$ has a simple zero at $\epsilon=0$ (see Appendix 5) one writes the following small $\epsilon$ expansion:

$$
\begin{aligned}
e^{2 \pi i \epsilon \alpha^{\prime}} & K_{n-r, m-s}\left(\alpha_{r, s}+\frac{\epsilon}{2}\right) \\
= & \frac{S_{b}\left(2 \alpha_{n, m}+\mu\right) S_{b}\left(2 \alpha_{n-r, m-s}+\mu\right)}{S_{b}^{\prime}\left(2 \alpha_{n+1, m+1}\right) S_{b}^{\prime}\left(2 \alpha_{n-r+1, m-s+1}\right)} \\
& \times\left(\frac{1}{\epsilon}+2 \pi i \alpha^{\prime}+\frac{S_{b}^{\prime}\left(2 \alpha_{n, m}+\mu\right)}{S_{b}\left(2 \alpha_{n, m}+\mu\right)}\right. \\
- & \left.\frac{1}{2} \frac{S_{b}^{\prime \prime}\left(2 \alpha_{n+1, m+1}\right)}{S_{b}^{\prime}\left(2 \alpha_{n+1, m+1}\right)}+O(\epsilon)\right) .
\end{aligned}
$$

Now we turn to

$$
\begin{aligned}
& K_{n, m}\left(\alpha_{-r,-s}-\frac{\epsilon}{2}\right) \\
& \quad=\frac{S_{b}\left(2 \alpha_{n-r, m-s}+\mu-\epsilon\right) S_{b}\left(2 \alpha_{n, m}+\mu\right)}{S_{b}\left(2 \alpha_{n-r+1, m-s+1}-\epsilon\right) S_{b}^{\prime}\left(2 \alpha_{n+1, m+1}\right)} .
\end{aligned}
$$

This term has different expansions depending on the balance of indices. If $n \geq r, m \geq s$ we have an expansion similar to (29),

$$
\begin{aligned}
e^{-2 \pi i \epsilon \alpha^{\prime}} K_{n, m}\left(\alpha_{-r,-s}-\frac{\epsilon}{2}\right) \\
=\frac{S_{b}\left(2 \alpha_{n-r, m-s}+\mu\right) S_{b}\left(2 \alpha_{n, m}+\mu\right)}{S_{b}^{\prime}\left(2 \alpha_{n-r+1, m-s+1}\right) S_{b}^{\prime}\left(2 \alpha_{n+1, m+1}\right)} \\
\quad \times\left(-\frac{1}{\epsilon}+2 \pi i \alpha^{\prime}+\frac{S^{\prime}\left(2 \alpha_{n-r, m-s}+\mu\right)}{S_{b}\left(2 \alpha_{n-r, m-s}+\mu\right)}\right. \\
\left.-\frac{1}{2} \frac{S_{b}^{\prime \prime}\left(2 \alpha_{n-r+1, m-s+1}\right)}{S_{b}^{\prime}\left(2 \alpha_{n-r+1, m-s+1}\right)}+O(\epsilon)\right) .
\end{aligned}
$$

We see that the sum of (29) and (31) is indeed regular at $\epsilon=0$ and given by

$$
\begin{aligned}
\mathcal{M}_{n, m}^{r, s}(0)= & \frac{S_{b}\left(2 \alpha_{n-r, m-s}+\mu\right) S_{b}\left(2 \alpha_{n, m}+\mu\right)}{S_{b}^{\prime}\left(2 \alpha_{n-r+1, m-s+1}\right) S_{b}^{\prime}\left(2 \alpha_{n+1, m+1}\right)} \\
& \times\left(4 \pi i \alpha^{\prime}+\frac{S_{b}^{\prime}\left(2 \alpha_{n, m}+\mu\right)}{S_{b}\left(2 \alpha_{n, m}+\mu\right)}+\frac{S_{b}^{\prime}\left(2 \alpha_{n-r, m-s}+\mu\right)}{S_{b}\left(2 \alpha_{n-r, m-s}+\mu\right)}\right. \\
& \left.-\frac{1}{2} \frac{S_{b}^{\prime \prime}\left(2 \alpha_{n+1, m+1}\right)}{S_{b}^{\prime}\left(2 \alpha_{n+1, m+1}\right)}-\frac{1}{2} \frac{S_{b}^{\prime \prime}\left(2 \alpha_{n-r+1, m-s+1}\right)}{S_{b}^{\prime}\left(2 \alpha_{n-r+1, m-s+1}\right)}\right), \\
& n \geq r, m \geq s .
\end{aligned}
$$

When $n<r$ and $m<s$ function $S_{b}^{-1}\left(2 \alpha_{n-r+1, m-s+1}-\epsilon\right)=$ $O(\epsilon)$ so the second term in (27) vanishes at $\epsilon=0$, while the first term is absent due to the factor $\delta_{n \geq r, m \geq s}$; hence

$\mathcal{M}_{n, m}^{r, s}(0)=0, \quad n<r, m<s$.

Finally, when $n<r$ and $m \geq s$ or $n \geq r$ and $m<s$ the function $S_{b}^{-1}\left(2 \alpha_{n-r+1, m-s+1}-\epsilon\right)$ is regular at $\epsilon=0$ and we have

$$
\begin{array}{r}
\mathcal{M}_{n, m}^{r, s}(0)=\frac{S_{b}\left(2 \alpha_{n-r, m-s}+\mu\right) S_{b}\left(2 \alpha_{n, m}+\mu\right)}{S_{b}\left(2 \alpha_{n-r+1, m-s+1}\right) S_{b}^{\prime}\left(2 \alpha_{n+1, m+1}\right)}, \\
\quad \times n<r, m \geq s \text { or } n \geq r, m<s .
\end{array}
$$

\subsubsection{Expansion near $\alpha=\alpha_{r,-s}$}

Now let us expand $\mathcal{M}_{\alpha \alpha^{\prime}}$ near $\alpha=\alpha_{r,-s}$ :

$$
\begin{aligned}
\mathcal{M}_{\alpha_{r,-s}+\frac{\epsilon}{2}, \alpha^{\prime}}= & e^{-4 \pi i \alpha_{r, s}}\left(e^{2 \pi i \epsilon \alpha^{\prime}} e^{8 \pi i \alpha_{r, 0} \alpha^{\prime}} K_{\alpha_{r, s}+\frac{\epsilon}{2}, \alpha^{\prime}}\right. \\
& \left.+e^{-2 \pi i \epsilon \alpha^{\prime}} e^{8 \pi i \alpha_{0, s} \alpha^{\prime}} K_{\alpha_{-r, s}-\frac{\epsilon}{2}, \alpha^{\prime}}\right) \\
= & e^{-4 \pi i \alpha_{r, s}} \sum_{n, m} e^{8 \pi i \alpha_{n, m} \alpha^{\prime}} \mathcal{M}_{n, m}^{r,-s}(\epsilon)
\end{aligned}
$$


where we have denoted

$$
\begin{aligned}
\mathcal{M}_{n, m}^{r,-s}(\epsilon)= & e^{2 \pi i \epsilon \alpha^{\prime}} K_{n-r, m}\left(\alpha_{r,-s}+\frac{\epsilon}{2}\right) \delta_{n \geq r} \\
& +e^{-2 \pi i \epsilon \alpha^{\prime}} K_{n, m-s}\left(\alpha_{-r, s}-\frac{\epsilon}{2}\right) \delta_{m \geq s} .
\end{aligned}
$$

We emphasize that $\mathcal{M}_{n, m}^{r,-s}(\epsilon)$ is not obtained from $\mathcal{M}_{n, m}^{r, s}(\epsilon)$ (27) by flipping the sign of $s$.

Proceeding in full analogy with the previous subsection one shows that $\mathcal{M}_{n, m}^{r,-s}(\epsilon)$ is regular at $\epsilon=0$ with different expansions depending on $n-r$ and $m-s$. For $n \geq r, m \geq s$ one obtains

$$
\begin{aligned}
\mathcal{M}_{n, m}^{r,-s}(0)= & \frac{S_{b}\left(2 \alpha_{n, m-s+\mu}\right) S_{b}\left(2 \alpha_{n-r, m+\mu}\right)}{S_{b}^{\prime}\left(2 \alpha_{n-r+1, m+1}\right) S_{b}^{\prime}\left(2 \alpha_{n+1, m-s+1}\right)} \\
& \times\left(4 \pi i \alpha^{\prime}+\frac{S_{b}^{\prime}\left(2 \alpha_{n-r, m}+\mu\right)}{S_{b}\left(2 \alpha_{n-r, m}+\mu\right)}\right. \\
& +\frac{S_{b}^{\prime}\left(2 \alpha_{n, m-s}+\mu\right)}{S_{b}\left(2 \alpha_{n, m-s}+\mu\right)}-\frac{1}{2} \frac{S_{b}^{\prime \prime}\left(2 \alpha_{n+1, m-s+1}\right)}{S_{b}^{\prime}\left(2 \alpha_{n+1, m-s+1}\right)} \\
& \left.-\frac{1}{2} \frac{S_{b}^{\prime \prime}\left(2 \alpha_{n-r+1, m+1}\right)}{S_{b}^{\prime}\left(2 \alpha_{n-r+1, m+1}\right)}\right), \quad n \geq r, m \geq s .
\end{aligned}
$$

When $n<r$ and $m<s \mathcal{M}_{n, m}^{r,-s}(0)$ is vanishing due, to the Kronecker deltas

$\mathcal{M}_{n, m}^{r,-s}(0)=0, \quad n<r, m<s$,

and finally when $n<r$ and $m \geq s$ or $n \geq r$ and $m<s$ there are no singular terms and one has

$$
\begin{aligned}
\mathcal{M}_{n, m}^{r,-s}(0) & =\frac{S_{b}\left(2 \alpha_{n-r, m}+\mu\right) S_{b}\left(2 \alpha_{n, m-s}+\mu\right)}{S_{b}\left(2 \alpha_{n-r+1, m+1}\right) S_{b}^{\prime}\left(2 \alpha_{n+1, m-s+1}\right)}, \\
n<r, m & \geq s, \\
\mathcal{M}_{n, m}^{r,-s}(0) & =\frac{S_{b}\left(2 \alpha_{n-r, m}+\mu\right) S_{b}\left(2 \alpha_{n, m-s}+\mu\right)}{S_{b}\left(2 \alpha_{n+1, m-s+1}\right) S_{b}^{\prime}\left(2 \alpha_{n-r+1, m+1}\right)}, \\
n \geq r, m & <s .
\end{aligned}
$$

\subsubsection{Comparison}

Let us first compare Eqs. (32) and (37), which are valid for $n \geq r, n \geq s$. Consider the ratio of $\mu$-dependent terms in the overall prefactors. Using property (56) one obtains

$$
\begin{aligned}
& \frac{S_{b}\left(2 \alpha_{n, m-s}+\mu\right) S_{b}\left(2 \alpha_{n-r, m}+\mu\right)}{S_{b}\left(2 \alpha_{n-r, m-s}+\mu\right) S_{b}\left(2 \alpha_{n, m}+\mu\right)} \\
& \quad=\frac{\prod_{k=n-r}^{n-1} 2 \sin \pi\left(k b^{2}+\mu b+m-s\right)}{\prod_{k=n-r}^{n-1} 2 \sin \pi\left(k b^{2}+\mu b+m\right)}=(-1)^{r s} .
\end{aligned}
$$

Now, differentiating (56), substituting $z=2 \alpha_{n+1, m+1}$, and taking into account that $S_{b}\left(2 \alpha_{n+1, m+1}\right)=0$ we have

$$
S_{b}^{\prime}\left(2 \alpha_{n+2, m+1}\right)=2 \sin 2 \pi b \alpha_{n, m} S_{b}^{\prime}\left(2 \alpha_{n+1, m+1}\right) .
$$

Using this equation one computes the ratio of the remaining $\mu$-independent terms in the overall prefactors of (32) and (37),

$$
\begin{aligned}
& \frac{S_{b}^{\prime}\left(2 \alpha_{n-r+1, m-s+1}\right) S_{b}^{\prime}\left(2 \alpha_{n+1, m+1}\right)}{S_{b}^{\prime}\left(2 \alpha_{n+1, m-s+1}\right) S_{b}^{\prime}\left(2 \alpha_{n-r+1, m+1}\right)} \\
& =\frac{\prod_{k=n-r+1}^{n} 2 \sin \pi\left(k b^{2}+m+1\right)}{\prod_{k=n-r+1}^{n} 2 \sin \pi\left(k b^{2}+m-s+1\right)}=(-1)^{r s} .
\end{aligned}
$$

Hence, the overall factors are the same in (32) and (37) and the $\alpha^{\prime}$-dependent terms agree exactly. Now, consider

$$
\begin{aligned}
T_{n, m}^{r, s} & =\frac{S_{b}^{\prime}\left(2 \alpha_{n-r, m-s}+\mu\right)}{S_{b}\left(2 \alpha_{n-r, m-s}+\mu\right)}+\frac{S_{b}^{\prime}\left(2 \alpha_{n, m}+\mu\right)}{S_{b}\left(2 \alpha_{n, m}+\mu\right)}, \\
T_{n, m}^{r,-s} & =\frac{S_{b}^{\prime}\left(2 \alpha_{n-r, m}+\mu\right)}{S_{b}\left(2 \alpha_{n-r, m}+\mu\right)}+\frac{S_{b}^{\prime}\left(2 \alpha_{n, m-s}+\mu\right)}{S_{b}\left(2 \alpha_{n, m-s}+\mu\right)},
\end{aligned}
$$

which enter Eqs. (32) and (37), respectively. We will show by induction in $r, s$ that these functions coincide. For $r, s=0$ this is trivial. Assume that $T_{n, m}^{r, s}=T_{n, m}^{r,-s}$ for some $r, s$ and consider

$$
\begin{aligned}
& T_{n, m}^{r+1, s} \\
& =\frac{S_{b}^{\prime}\left(2 \alpha_{n-r+1, m-s}+\mu\right)}{S_{b}\left(2 \alpha_{n-r-1, m-s}+\mu\right)}+\cdots=\frac{2 \sin \pi b\left(2 \alpha_{n-r, m-s}+\mu\right)}{S_{b}\left(2 \alpha_{n-r, m-s}+\mu\right)} \\
& \quad \times \frac{S_{b}^{\prime}\left(2 \alpha_{n-r, m-s}+\mu\right)-2 \pi b \cos \pi b\left(2 \alpha_{n-r-1, m-s}+\mu\right) S_{b}\left(2 \alpha_{n-r-1, m-s}+\mu\right)}{2 \sin \pi b\left(2 \alpha_{n-r, m-s}+\mu\right)} \\
& \quad+\cdots=T_{n, m}^{r, s}-\pi b \cot \pi\left((n-r-1) b^{2}+\mu b\right)
\end{aligned}
$$

where in the intermediate steps the $r$-independent part of $T_{n, m}^{r, s}$ is denoted by ellipses. Also, besides Eq. (56) we have used the relation

$S_{b}^{\prime}(z+b)=2 \sin \pi b z S_{b}^{\prime}(z)+2 \pi b \cos \pi b z S_{b}(z)$,

which is obtained by differentiating (56). Mimicking the above computation one shows that

$T_{n, m}^{r+1,-s}=T_{n, m}^{r,-s}-\pi b \cot \pi\left((n-r-1) b^{2}+\mu b\right)$.

Hence $T_{n, m}^{r+1, s}=T_{n, m}^{r+1,-s}$. Induction in $s$ proceeds in full analogy and we will omit it.

A last step in verifying agreement between (32) and (37) is to show that the functions

$$
\begin{aligned}
U_{n, m}^{r, s} & =\frac{S_{b}^{\prime \prime}\left(2 \alpha_{n-r+1, m-s+1}\right)}{S_{b}^{\prime}\left(2 \alpha_{n-r+1, m-s+1}\right)}+\frac{S_{b}^{\prime \prime}\left(2 \alpha_{n+1, m+1}\right)}{S_{b}^{\prime}\left(2 \alpha_{n+1, m+1}\right)}, \\
U_{n, m}^{r,-s} & =\frac{S_{b}^{\prime \prime}\left(2 \alpha_{n-r+1, m+1}\right)}{S_{b}^{\prime}\left(2 \alpha_{n-r+1, m+1}\right)}+\frac{S_{b}^{\prime \prime}\left(2 \alpha_{n+1, m-s+1}\right)}{S_{b}^{\prime}\left(2 \alpha_{n+1, m-s+1}\right)},
\end{aligned}
$$


also coincide. We confine ourselves to pointing out that the property

$$
\begin{aligned}
S_{b}^{\prime \prime}\left(2 \alpha_{n+2, m+1}\right)= & 2 \sin 2 \pi b \alpha_{n+1, m+1} S_{b}^{\prime \prime}\left(2 \alpha_{n+1, m+1}\right) \\
& +4 \pi b \cos 2 \pi b \alpha_{n+1, m+1} S_{b}^{\prime}\left(2 \alpha_{n+1, m+1}\right)
\end{aligned}
$$

reduces the comparison of $U_{n, m}^{r, s}$ and $U_{n, m}^{r,-s}$ to basically the same computation as we carried out for $T_{n, m}^{r, \pm s}$ and we will not present it here. Equation (51) is obtained from (56) by double differentiation and substitution of $z=2 \alpha_{n+1, m+1}$ together with using $S_{b}\left(2 \alpha_{n+1, m+1}\right)=0$.

Hence we have shown that

$\mathcal{M}_{n, m}^{r, s}(0)=\mathcal{M}_{n, m}^{r,-s}(0), \quad n \geq r, m \geq s$.

Coincidence of these functions for $n<r, m<s$ is trivial since they both vanish; see Eqs. (33) and (38). It remains to compare (34) with (39) and (40). This is again a straightforward but somewhat bulky exercise making use of Eqs. (56) and (42). We will omit the computation and only report complete agreement. This completes our proof of Eq. (20).

\section{Discussion}

The non-perturbative aspects of $\mathrm{CBs}$ are hard to reveal. Zamolodchikov's formula (4), describing the analytic structure of $\mathrm{CB}$ to all orders in $q$, is a remarkable exception. The fact that the explicit expression for the modular kernel of generic CB is available (14) is also quite non-trivial. It is instructive to recall how this expression is derived $[11,12]$. The algebra of modular transformations features non-linear consistency relations such as the pentagon and the hexagon identities and their toric counterparts [13]. Certain specifications of these non-linear relations give rise to linear difference equations on the generic modular kernel with degenerate modular kernels entering as coefficients. Degenerate CBs correspond to finite representations of the Virasoro algebra and satisfy the differential BPZ equations. They can be found exactly and the corresponding modular kernels (which are simply finite matrices) can be computed. Hence, in deriving Eq. (18) only properties of a very special class of CBs was explicitly used, but the result is supposed to describe the modular transformations of generic CB. The validity of Eq. (10) following from the analytic structure of generic $\mathrm{CB}$ furnishes a highly non-trivial test of this assertion.

Moreover, these equations partly explain an unexpected structure of Eq. (18). As confirmed from many perspectives $[12,14-19]$ the Fourier-type contribution $e^{4 \pi i \alpha \alpha^{\prime}}$ is always present in the modular kernel at the perturbative level. ${ }^{4}$

\footnotetext{
${ }^{4}$ Here the perturbative expansion in inverse powers of $\Delta$ as $\Delta \rightarrow \infty$ is implied. This should not be confused with the perturbative $q$-expansion which we usually discuss in the text.
}

From this point of view, the expansion in (18) looks like a non-perturbative completion with powers of parameters $e^{4 \pi i b \alpha}, e^{4 \pi i b^{-1} \alpha}$ which do not appear in $q$-expansion of CB. Equation (10), valid on general grounds, cannot be satisfied by the Fourier kernel alone and necessitates the introduction of the aforementioned non-perturbative terms.

One more remark is in order. Zamolodchikov's relation for $\mathrm{CB}$ is powerful enough to replace the definition and give an efficient computational approach. Although we already have an explicit formula for the modular kernel it is interesting to understand whether a relation similar to Zamolodchikov's recursion can be found for the modular kernel based solely on property (10). We argue that this is not the case. It is the non-trivial series expansion part of the modular kernel $\mathcal{M}_{\alpha \alpha^{\prime}}$ (17) for which we would like to obtain a recursive definition. However, this part is regular and only satisfies condition (20). This is not enough to construct a recurrence equation valid for all $\alpha$. In other words, in the full modular kernel $M_{\alpha \alpha^{\prime}}$ (14) all the poles come from an $\alpha^{\prime}$-independent normalization factor $V_{\alpha}$, and hence they are common to all coefficients of the expansion that we wish to describe. In contrast, in the expansion of the conformal block (2) additional poles appear as the order of $q$ increases. This is the reason why (3) relates different orders of the $q$-expansion allowing for recursive computations.

Finally, we would like to stress that although we have only checked Eq. (8) for the toric Virasoro block; the derivation is very general and extensions to many other cases should exist. For example, the spheric modular kernel should satisfy (8) if the toric residue coefficients $R_{r, s}$ are replaced by their spheric counterparts.

Acknowledgements The author is grateful to Alexei Morozov and Andrey Mironov for their guidance. The work is partly supported by Grants RFBR 16-01-00291, RFBR 16-32-00920-mol-a, RFBR 15-5152031-NSC-a, RFBR 16-51-53034-GFEN, RFBR 15-51-50034-YaF, and MK-8769.2016.1. The author gratefully acknowledges the financial support of the Ministry of Education and Science of the Russian Federation in the framework of Increase Competitiveness Program of MISIS.

Open Access This article is distributed under the terms of the Creative Commons Attribution 4.0 International License (http://creativecomm ons.org/licenses/by/4.0/), which permits unrestricted use, distribution, and reproduction in any medium, provided you give appropriate credit to the original author(s) and the source, provide a link to the Creative Commons license, and indicate if changes were made.

Funded by SCOAP ${ }^{3}$.

\section{Double gamma and sine functions}

The double gamma function $\Gamma_{b}(z)$ can be defined as the analytic continuation of the following integral: 


$$
\begin{aligned}
\Gamma_{b}(z)= & \int_{0}^{\infty} \frac{\mathrm{d} t}{t}\left(\frac{e^{-z t}-e^{-Q t / 2}}{\left(1-e^{-b t}\right)\left(1-e^{-b^{-1} t}\right)}-\frac{(Q-2 z)^{2}}{8 e^{t}}\right. \\
& \left.-\frac{Q-2 z}{2 t}\right), \\
Q= & b+b^{-1} .
\end{aligned}
$$

$\Gamma_{b}(z)$ is meromorphic with no zeros and only simple poles located at $z=-n b-m b^{-1}$ for $n, m \geq 0$, i.e. schematically $\Gamma_{b}(z) \propto \prod_{n, m \geq 0} \frac{1}{z+r b+s b^{-1}}$. The double gamma function satisfies the following difference equations:

$$
\begin{gathered}
\Gamma_{b}(z+b)=\Gamma_{b}(z) \frac{\sqrt{2 \pi} b^{b z-1 / 2}}{\Gamma(b z)}, \\
\Gamma_{b}\left(z+b^{-1}\right)=\Gamma_{b}(z) \frac{\sqrt{2 \pi} b^{1 / 2-b^{-1} z}}{\Gamma\left(b^{-1} z\right)},
\end{gathered}
$$

related to each other by the replacement $b \rightarrow b^{-1}$, which is a symmetry of the double gamma function, $\Gamma_{b}(z)=\Gamma_{b^{-1}}(z)$.

The double sine function $S_{b}(z)$ is defined as

$$
S_{b}(z)=\frac{\Gamma_{b}(z)}{\Gamma_{b}(Q-z)} \text {. }
$$

It shares poles with the double gamma function but possesses additional zeros at $z=n b+m b^{-1}$ for $n, m \geq 1$, i.e. schematically $S_{b}(z) \propto \prod_{n, m \geq 0} \frac{z-(n+1) b-(m+1) b^{-1}}{z+n b+m b}$. The double sine function satisfies the following difference equations:

$$
\begin{aligned}
S_{b}(z+b) & =2 \sin (\pi b z) S_{b}(z), \quad S_{b}\left(z+b^{-1}\right) \\
& =2 \sin \left(\pi b^{-1} z\right) S_{b}^{-1}(z)
\end{aligned}
$$

and the symmetry property $S_{b}(z)=S_{b^{-1}}(z)$.

\section{References}

1. A. Belavin, A. Polyakov, A. Zamolodchikov, Infinite conformal symmetry in two-dimensional quantum field theory. Nucl. Phys. B 241, 333-380 (1984). doi:10.1016/0550-3213(84)90052-X

2. L. Alday, D. Gaiotto, Y. Tachikawa, Liouville correlation functions from four-dimensional gauge theories. Lett. Math. Phys. 91, 167197 (2010). doi:10.1007/s11005-010-0369-5. arXiv:0906.3219 [hep-th]

3. N. Wyllard, A(N-1) conformal Toda field theory correlation functions from conformal $\mathrm{N}=2 \mathrm{SU}(\mathrm{N})$ quiver gauge theories. JHEP 0911, 002 (2009). doi:10.1088/1126-6708/2009/11/002. arXiv:0907.2189 [hep-th]
4. A. Mironov, A. Morozov, On AGT relation in the case of U(3). Nucl. Phys. B 825, 1-37 (2010). doi:10.1016/j.nuclphysb.2009. 09.011. arXiv:0908.2569 [hep-th]

5. E. Perlmutter, Virasoro conformal blocks in closed form. JHEP $\mathbf{0 8}$, 088 (2015). doi:10.1007/JHEP08(2015)088. arXiv:1502.07742 [hep-th]

6. Al Zamolodchikov, Conformal symmetry in two-dimensional space. Theor. Math. Phys. 73, 1088-1093 (1987)

7. Al. Zamolodchikov, "Conformal symmetry in two dimensions: an explicit recurrence formula for the conformal partial wave amplitude. Commun. Math. Phys. 96, 419-422 (1984). doi:10.1007/ BF01214585

8. V.A. Fateev, A.V. Litvinov, On AGT conjecture. JHEP 02, 014 (2010). doi:10.1007/JHEP02(2010)014. arXiv:0912.0504 [hep-th]

9. R. Poghossian, Recursion relations in CFT and N=2 SYM theory. JHEP 12, 038 (2009). doi:10.1088/1126-6708/2009/12/038. arXiv:0909.3412 [hep-th]

10. L. Hadasz, Z. Jaskolski, P. Suchanek, Recursive representation of the torus 1-point conformal block. JHEP 1001, 063 (2010). doi:10. 1007/JHEP01(2010)063. arXiv:0911.2353 [hep-th]

11. J. Teschner, From Liouville theory to the quantum geometry of Riemann surfaces. arXiv:hep-th/0308031 [hep-th]

12. N. Nemkov, On modular transformations of toric conformal blocks. JHEP 10, 039 (2015). doi:10.1007/JHEP10(2015)039. arXiv:1504.04360 [hep-th]

13. G. Moore, N. Seiberg, Lectures on RCFT. RU-89-32, YCTP-P1389, C89-08-14 (1989)

14. D. Galakhov, A. Mironov, A. Morozov, S-duality as a betadeformed Fourier transform. JHEP 1208, 067 (2012). doi:10.1007/ JHEP08(2012)067. arXiv:1205.4998 [hep-th]

15. N. Nemkov, S-duality as Fourier transform for arbitrary $\epsilon_{1}, \epsilon_{2}$. J. Phys. A Math. Theor. 47, 105401 (2014). doi:10.1088/1751-8113/ 47/10/105401. arXiv:1307.0773 [hep-th]

16. M. Billo, M. Frau, L. Gallot, A. Lerda, I. Pesando, Modular anomaly equation, heat kernel and S-duality in $N=2$ theories. JHEP 1311, 123 (2013). doi:10.1007/JHEP11(2013)123. arXiv:1307.6648 [hep-th]

17. M. Billo, M. Frau, L. Gallot, A. Lerda, I. Pesando, Deformed N=2 theories, generalized recursion relations and S-duality. JHEP 1304, 039 (2013). doi:10.1007/JHEP04(2013)039. arXiv:1302.0686 [hep-th]

18. D. Galakhov, A. Mironov, A. Morozov, S-Duality and modular transformation as a non-perturbative deformation of the ordinary pq-duality. JHEP 06, 050 (2014). doi:10.1007/JHEP06(2014)050. arXiv:1311.7069 [hep-th]

19. N. Nemkov, On fusion kernel in Liouville theory. Theor. Math. Phys. 189(2), 1574-1591 (2016). doi:10.4213/tmf9137. arXiv:1409.3537 [hep-th] 